Mitteilungen der Österreichischen Geographischen Gesellschaft, 161. Jg., S. 395-397

(Annals of the Austrian Geographical Society, Vol. 161, pp. 395-397)

Wien (Vienna) 2019, https://doi.org/10.1553/moegg161s395

\title{
1. (NEUE) UNGEGN-Sitzung, New York, 29. APRIL - 3. MAI 2019
}

\author{
Peter JORDAN, Wien* \\ mit 2 Abb. im Text
}

Die diesjährige Sitzung der Expertengruppe der Vereinten Nationen für Geographische Namen („United Nations Group of Experts on Geographical Names“, UNGEGN) war eigentlich die 31. der seit 1967 stattfindenden Sitzungen, aber die erste nach dem neuen Organisationsmodell. In diesem wurden die Funktionen der Konferenzen der Vereinten Nationen zur Standardisierung Geographischer Namen und der bisherigen UNGEGN-Sitzungen zusammengelegt. Zum ersten Mal hatte nun eine UNGEGN-Sitzung die Kompetenz, auch Resolutionen der Vereinten Nationen zu verabschieden, die dann nur noch vom Wirtschafts- und Sozialrat der Vereinten Nationen („UN Economic and Social Council“, ECOSOC) bestätigt werden müssen. Mit dieser Zusammenlegung reagierte man auf die Anforderungen der Kooperation mit der Expertenkommission zum Globalen Georäumlichen Informationsmanagement der Vereinten Nationen („Committee of Experts on Global Geospatial Information Management“, UN-GGIM), die 2011 ebenfalls unter dem Dach des ECOSOC eingerichtet worden war. Wie jene der UN-GGIM finden auch die UNGEGN-Sitzungen alle zwei Jahre statt.

Im praktischen Ablauf erwies sich die neue UNGEGN-Sitzung aber als den vorhergegangenen 30 Sitzungen sehr ähnlich. Auch Befürchtungen, sie würde mehr politischen als fachlichen Diskussionen gewidmet sein, bewahrheiteten sich nicht. Wie üblich wurden Berichte von Staaten, den sprachlichen oder geographischen Staatengruppen („Divisions“), der thematischen Arbeitsgruppen („Working Groups") und von Organisationen, mit denen die UNGEGN in Verbindung steht, von wissenschaftlichen Vorträgen und Diskussionen begleitet, die wie immer von der Tatsache profitierten, dass sich die UNGEGN aus Experten aller Wissenschaften mit Relevanz für geographische Namen zusammensetzt, also aus Linguisten, Historikern, Geographen, Kartographen, Geodäten, Kulturanthropologen und Rechtswissenschaftlern. Sie bildet damit ein Forum für eine umfassende Diskussion über geographische Namen, allerdings mit einem Schwerpunkt auf Fragen der Standardisierung und mit einer starken Betonung der kulturellen Perspektive, welche die in Konfliktsituationen so deutlich erkennbare symbolische Wirkung von geographischen Namen hervorhebt.

An der Sitzung nahmen 254 Delegierte aus 64 Staaten teil, wobei Indonesien (26 Teilnehmer), China (12), die Republik Korea (12) und Japan (10) die größten Delegationen stellten. Außerdem waren etliche internationale Organisationen vertreten, so die Internationale Geographische Union („International Geographical Union“, IGU), der Internationale Rat der Onomastischen Wissenschaften („International Council of Onomastic Sciences“, ICOS), die Internationale Hydrographische Organisation („International Hydrographic Organization“, IHO) und die Internationale Kartographische Vereinigung („International Cartographic Association“, ICA). Die IGU wurde von Cosimo PALA-

\footnotetext{
* Hofrat i.R. Prof. h.c. Univ.-Doz. Dr. Peter Jordan, Institut für Stadt- und Regionalforschung, Österreichische Akademie der Wissenschaften; Research Fellow, University of the Free State Bloemfontein, Faculty of the Humanities, South Africa; Postgasse 7/4/2, A-1010 Wien; E-Mail: peter.jordan@oeaw.ac.at
} 
GIANO (Italien) vertreten, die ICA vom Berichterstatter, der in der Funktion eines „ICA Liaison Officers" Ferjan Ormeling nachgefolgt war (Abb. 1). Peter JoRDAn präsentierte im Plenum auch den Bericht der „Joint ICA/IGU Commission on Toponymy“ und sprach in einer Begleitveranstaltung dieser Kommission über die aktuellen geographischen und kartographischen Hauptforschungsrichtungen in der Toponomastik.



Foto: $\quad$ Matjaž GERŠIČ, 2019

Abb. 1: Peter Jordan und Adriana Vescovo (Argentinien) als „Liaison Officer“ bzw. Beobachterin der „International Cartographic Association“ (ICA) bei der UNGEGN-Sitzung 2019 in New York

Die österreichische Delegation setzte sich aus dem Innsbrucker Linguisten Gerhard RAMPL, der sie als neuer Vorsitzender der „Arbeitsgemeinschaft für Kartographische Ortsnamenkunde“ (AKO) auch erstmals leitete, und Peter JoRDAN zusammen. RAmPL trug als internationaler Koordinator der „Toponymic Guidelines for Map and Other Editors“ auch den entsprechenden Bericht vor. JoRDAN sprach im Plenum zum Thema „The border between ,ours“ and ,theirs ' drawn by place names“1) und beleuchtete damit einen Wesenskern der Unterscheidung zwischen Endonymen und Exonymen.

Ansonsten kreisten die Vorträge und Diskussionen sehr stark um das Thema „Geographische Namen als kulturelles Erbe“, was die kritischen Fragen von kommerziellen Namen und Gedenknamen, die manchmal traditionelle Namen ersetzen, einschloss, sowie um Namen in Städten (Namen von Verkehrsflächen), wiederum mit Überlegungen, wie man kommerzielle und Gedenknamen vermeiden könnte. Am eindrucksvollsten war in diesem Zusammenhang ein Beitrag von Ingvil NoRDLAND aus Norwegen, die die norwegische Sprach- und Ortsnamenpolitik beleuchtete und zeigte, dass sich hier allgemein akzeptierte Grundsätze teilweise widersprechen. Sie stellte auch die Frage, für wen wir denn eigentlich Standardisierung betreiben: für die lokale und regionale Gemeinschaft (dann vielleicht am besten durch die Standardisierung von geographischen Namen in ihrer Dialektform), für die nationale oder für die internationale Gemeinschaft?

\footnotetext{
1) https://unstats.un.org/unsd/ungegn/sessions/2019-new-york-ungegn-1st-session/documents/GEGN.2 2019_62_CRP.62_Exonyms.pdf
} 
Sonderpräsentationen von Experten außerhalb der UNGEGN erweiterten wie gewohnt den wissenschaftlichen Rahmen. Von den vier Beiträgen erfuhr der Vortrag „Indigene Fragen und kulturelles Erbe“ eines Vertreters des Ständigen Forums für Indigene Fragen („Permanent Forum on Indigenous Issues“) die größte Resonanz. Er beschrieb die wachsende Repräsentanz der Namen aus Minderheitensprachen im Nordosten der Ostsee (Estland, Leningrader Gebiet [Leningradskaja oblast ${ }^{`}$ ], Karelien [Karelija/Karjala] im öffentlichen Raum und zeigte, dass Bewegungen, die dieses Ziel unterstützen, in hohem Maß von jungen Menschen getragen werden und fast Teil einer Jugendkultur sind. Er wies auch darauf hin, dass Namen aus Minderheitensprachen im öffentlichen Raum ein erster Schritt auf dem Weg zur Wiederbelebung bereits verklungener oder nahezu verklungener Sprachen sein können, da die Repräsentanz der Namen im öffentlichen Raum nicht nur ein Anerkennen der Namen, sondern auch der zugehörigen Sprache bedeutet. Das Gefühl dieser Anerkennung macht Menschen eher geneigt, diese Sprache zu erlernen und zu verwenden.

Eine weitere Sonderpräsentation befasste sich mit dem Programm „Grenzen der Zweiten Verwaltungsebene (,Second Administrative Level Boundaries“, SALB), das von der Abteilung für Geoinformation und der Abteilung für Statistik der Vereinten Nationen durchgeführt wurde.

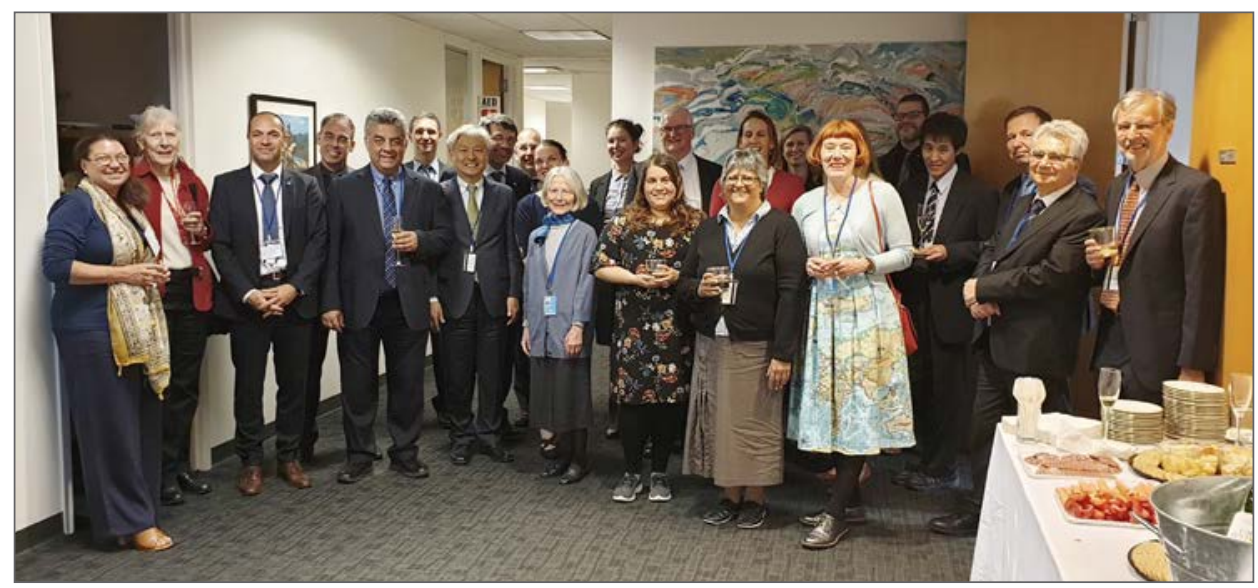

Zweite von links Helen Kerfoot (Kanada), die langjährige UNGEGN-Vorsitzende, dritter von links Pier-Giorgio ZACCHEDDU (Deutschland), 6. von links Gerhard RAMPL, Leiter der österreichischen Delegation, 7. von links Sungjae Сноо (Republik Korea), neuer stellvertretender UNGEGN-Vorsitzender, 14. von links (ganz hinten) William WaTt (Australien), scheidender UNGEGN-Vorsitzender, 15. von links Wendy SHAw (Neuseeland), neue Rapporteurin, zweiter von rechts Cosimo Palagiano (Italien), IGU-Liaison Officer, erster von rechts Peter JoRDAN, ICA-Liaison Officer

Foto: $\quad$ Matjaž GeRšIČ, 2019

Abb. 2: Der Führungskreis der UNGEGN bei der Verabschiedung von William WATT als UNGEGNVorsitzender in der australischen Gesandtschaft in New York

Die Sitzung wurde mit einer Geste des Dankes für den scheidenden UNGEGN-Vorsitzenden William WATT (Australien) abgeschlossen (Abb. 2), der den kritischen Übergang von der alten zur neuen UNGEGN so erfolgreich gemeistert hatte, sowie mit der Wahl des neuen Präsidiums mit Pierre JAILLARD (Frankreich) als neuem UNGEGN-Vorsitzenden an der Spitze, Hasanuddin Z. ABIDIN (Indonesien) und Sungjae Chоо (Republik Korea) als stellvertretenden Vorsitzenden sowie Trent PALMER (USA) und Wendy SHAw (Neuseeland) als Rapporteuren. 